\title{
Comparison of Sliding Mode Controller and Linear Quadratic Regulator (LQR) on a Two-Wheel Inverted Pendulum Robot: Design and Experiments
}

\author{
Mikail S. Arani \\ Department of Mechanical, \\ Industrial \& Aerospace \\ Engineering Concordia \\ University \\ Montreal, QC, Canada \\ m_shapoo@encs.concordia.
}

ca

\author{
Hamid Ebrahimi Orimi \\ Department of Mechanical, \\ Industrial \& Aerospace \\ Engineering Concordia \\ University \\ Montreal, QC, Canada \\ Ha_ebrah@encs.concordia.ca
}

\author{
Wen-Fang Xie \\ Department of Mechanical, \\ Industrial \& Aerospace \\ Engineering \\ Concordia University \\ Montreal, QC, Canada \\ wfxie@encs.concordia.ca
}

\author{
Henry Hong \\ Department of Mechanical, \\ Industrial \& Aerospace \\ Engineering \\ Concordia University \\ Montreal, QC, Canada \\ henry.hong@concordia.ca
}

\begin{abstract}
The current paper investigates two control methods for stabilizing a two-wheel inverted pendulum (TWIP) robot. It is well-known that the controller in the mobile robot plays a critical role in self-balancing and stabilizing. The TWIP robot has two DC gear motors with a high-resolution encoder and zero backlash, but with friction. It is a highly nonlinear and unstable system, which poses challenges for controller design. In this paper, a mathematical dynamic model is built using Lagrangian function method. Furthermore, a sliding mode controller (SMC) is proposed for auto-balancing and yaw rotation. A comparison of the proposed SMC controller and linear quadratic regulator (LQR) has been conducted. The experimental test results demonstrate the SMC controller outperforms (LQR) in terms of transient performance and disturbance rejection ability.
\end{abstract}

Keywords- Linear Quadratic Regulator (LQR), Sensor data fusion, Sliding mode control, Two-wheel inverted pendulum (TWIP) robot

\section{INTRODUCTION}

In recent years, self-balancing robots have attracted increasing attention in both industry and academia, since designing and testing control algorithms become more achievable with the rapid development of microcontrollers. Two-wheeled inverted pendulum (TWIP) robots have become more and more popular due to its light weight, small footprint, rapid rotation and high maneuverability. One of the applications of using these robots can be a service robot platform like Segway. However, the inherent instability and nonlinearity of the TWIP requires a sophisticated control scheme.

PID is a commonly used non-model based control method for self-balance robot, as there is no need to build the mathematics model. The three parameters can be tuned by trial and error or by experience. Although PID owns some robustness to disturbance and uncertainties to a certain extent, it cannot handle the sudden, large disturbance and modeling error [1]. Moreover, the control input cannot perfectly be optimized by using PID control. It could be targeted by having state feedback control (SFC) with optimized linear quadratic regulator (LQR) [2]. However, the optimized SFC can only work in the certain operating range and cannot react to system uncertainty. When the operating point is changed, the control gains have to be re-tuned in order to stabilize the robot, which is not very practical in the operation. Recently, the soft computing techniques such as fuzzy logic and neural networks have been used to control TWIP robot [3]. Two fuzzy controllers based on Mamdani and Takagi-Sugeno are designed for an inverted pendulum subjected to disturbance. Moreover, artificial neural network-based real-time switching dynamic controller is designed to solve balancing problem on various loose surfaces such as sand, pebble and soil [4]. A visual robot control interface is developed in C++ software development environment so that robot controller parameters can be changed as desired.

Recently, the model-based control of TWIP has been proposed to deal with the nonlinearity and disturbance. In [6], a linear SFC has been designed based on the linearized model, which needs very accurate dynamic models of the robots. One of the typical model-based control is SMC method. SMC is known to have a good robustness to the model uncertainties and disturbance. It can deal with the nonlinear unstable system with easy implementation, insensitivity to parameters variation and quick response independence of external disturbances. SMC has been successfully used for controlling the TWIP robot. In [5], a back-stepping slide model control has been proposed for balancing and trajectory tracking of a twowheeled robot. The balancing and angular velocity controllers are implemented in a cascade way.

In this paper, a dynamic model of a customer built TWIP robot is derived by using Lagrangian function method. The robot is composed of two gear DC motors driving two wheels, a structure, battery, sensors and controller. It is a highly nonlinear and unstable system. Two controllers, i.e. LQR and SMC have been designed for balancing and stabilizing the robot. Both controllers have been implemented on the robot and experimental tests have been carried out to compare performances of two controllers. The results demonstrate the 
superiority of SMC controller in terms of transient performance and disturbance rejection capability.

The paper is organized as follows. The paper begins with an introduction and the next section provides a dynamic of the model of the TWIP robot built in our lab. In Section III, the LQR and SMC are designed and the simulation results of both controllers are presented. Section IV presents the experiment results of both controllers. The conclusion is drawn in Section V.

\section{DYNAMIC MODELLING}

The TWIP robot is built in our lab to serve as test platform for various controllers. In order to control this type of unstable robot effectively, the first step is to model the robots dynamic behavior in the yaw and pitch motions. The schematic diagram of the TWIP robot is shown in Figure 1 and the parameters' description has been provided in TABLE 1 .

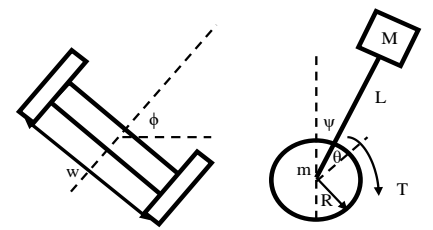

Figure. 1 Schematic diagram of the TWIP robot.

The dynamic model of the robot is built based on the Lagrangian function method [6]. Equation (1) presents Lagrangian where $\mathrm{L}_{1}, \mathrm{~L}_{2}$ and $\mathrm{B}$ are translation kinematic energy, rotational kinematic energy and potential energy, respectively.

$$
L_{a}=L_{1}+L_{2}+B
$$

It is assumed that the robot has 3 degree of freedoms (DOFs). The generalized variables of the robot are angle of the wheel $(\theta)$, pitch angle $(\phi)$ and yaw angle $(\psi)$ [7]. According to the robot dynamics, the translational and rotational kinetic energies are described as follows:

$$
\begin{gathered}
L_{1}=\frac{1}{2} m\left(\dot{x}_{l}{ }^{2}+\dot{y}_{l}{ }_{l}\right)+\frac{1}{2} m\left(\dot{x}_{r}^{2}+\dot{y}_{r}^{2}\right)+\frac{1}{2} M\left(\dot{x}_{b}{ }^{2}+\dot{y}_{b}{ }^{2}+\dot{z}_{b}{ }^{2}\right) \\
L_{2}=\frac{1}{2} J_{w} \dot{\theta}^{2}{ }_{l}+\frac{1}{2} J_{w} \dot{\theta}^{2}{ }_{r}+\frac{1}{2} J_{\psi} \dot{\psi}^{2}+\frac{1}{2} J_{\phi} \dot{\phi}^{2}{ }_{l}+\frac{1}{2} n^{2} J_{m}\left(\dot{\theta}_{l}-\dot{\psi}\right)^{2}+\frac{1}{2} n^{2} J_{m}\left(\dot{\theta}_{r}-\psi\right)^{2} \\
B=M g z_{b}
\end{gathered}
$$

The Lagrangian equations are:

$$
\begin{aligned}
& \frac{d}{d l}\left(\frac{\partial L_{a}}{\partial \dot{\theta}}\right)-\frac{\partial L_{a}}{\partial \theta}=F_{l}+F_{r} \\
& \frac{d}{d l}\left(\frac{\partial L_{a}}{\partial \dot{\psi}}\right)-\frac{\partial L_{a}}{\partial \psi}=-F_{l}-F_{r} \\
& \frac{d}{d l}\left(\frac{\partial L_{a}}{\partial \dot{\phi}}\right)-\frac{\partial L_{a}}{\partial \phi}=\frac{W}{2 R}\left(F_{r}-F_{l}\right)
\end{aligned}
$$

\begin{tabular}{|c|c|c|}
\hline Parameters & Unit & Description \\
\hline $\mathrm{W}=0.242$ & $m$ & Body Width \\
\hline $\mathrm{M}=1.047$ & $k g$ & Body Mass \\
\hline $\mathrm{m}=0.118$ & $\mathrm{~kg}$ & Wheels mass \\
\hline $\mathrm{R}=0.060$ & $m$ & Diameter of wheel \\
\hline $\mathrm{g}=9.810$ & $\mathrm{~m} / \mathrm{s}^{2}$ & Gravity acceleration \\
\hline $\mathrm{L}=0.030$ & $m$ & $\begin{array}{l}\text { Distance of the center of } \\
\text { the mass from the Wheel } \\
\text { axle }\end{array}$ \\
\hline $\mathrm{D}=0.050$ & $m$ & Body Depth \\
\hline $\mathrm{n}=64: 1$ & \multicolumn{2}{|c|}{ Gear ratio } \\
\hline $\mathrm{J}_{\mathrm{w}}=\mathrm{mR}^{2} / 2$ & $\mathrm{kgm}^{2}$ & Wheel inertia moment \\
\hline $\mathrm{J}_{\Psi}=\mathrm{ML}^{2} / 2$ & $\mathrm{kgm}^{2}$ & Body pitch inertia moment \\
\hline $\mathrm{J}_{\phi}=\mathrm{M}\left(\mathrm{W}^{2}+\mathrm{D}^{2}\right) / 12$ & $\mathrm{kgm}^{2}$ & Body yaw inertia moment \\
\hline $\mathrm{R}_{\mathrm{m}}=1.900$ & $\Omega$ & DC motor resistance \\
\hline $\mathrm{K}_{\mathrm{t}}=13.400$ & $m N m / A$ & DC motor torque constant \\
\hline $\mathrm{K}_{\mathrm{b}}=1.400$ & $m v / r p m$ & $\begin{array}{l}\text { DC motor back E.M.F } \\
\text { constant }\end{array}$ \\
\hline $\mathrm{fm}=0.0022$ & \multicolumn{2}{|c|}{$\begin{array}{l}\text { Friction coefficient between body } \\
\text { and DC motor }\end{array}$} \\
\hline$\theta, \psi, \phi$ & \multicolumn{2}{|c|}{$\begin{array}{l}\text { Rotary angle of the wheel, pitch angle } \\
\text { and yaw angle of the robot }\end{array}$} \\
\hline $1, \mathrm{r}, \mathrm{b}$ & \multicolumn{2}{|c|}{$\begin{array}{l}\text { Subscripts indicating left or right wheels } \\
\text { and the robot body, respectively }\end{array}$} \\
\hline
\end{tabular}

where, $F_{l}$ and $F_{r}$ are the torques on the left and right wheels, respectively. Moreover, they could be defined as follows:

$$
\begin{aligned}
& F_{l}=n K_{t} i_{l}+T_{s}+\sigma_{m}\left(\dot{\psi}-\dot{\theta}_{l}\right) \\
& F_{l}=n K_{t} i_{r}+T_{s}+\sigma_{m}\left(\dot{\psi}-\dot{\theta}_{r}\right)
\end{aligned}
$$

TABLE 1. Parameters of Two Wheeled Inverted Pendulum

By substituting the kinetic and potential energies in the Lagrangian equations:

$$
\begin{aligned}
& \left((2 m+M) R^{2}+2 J_{w}+2 n^{2} J_{m}\right) \ddot{\theta}+ \\
& \left(M R L \cos \psi-2 n^{2} J_{m}\right) \ddot{\psi}-M L R \psi^{2} \sin \psi=F_{\theta} \\
& \left(M R L \cos \psi-2 n^{2} J_{m}\right) \ddot{\theta}+\left(M L 2+J_{\psi}+2 n^{2} J_{m}\right) \ddot{\psi} \\
& -M g L \sin \psi-M L^{2} \dot{\phi}^{2} \sin \psi \cos \psi=F_{\psi} \\
& \left(0.5 m W^{2}+J J_{\phi}+\frac{W^{2}}{2 R^{2}}\left(J_{w}+n^{2} J_{m}\right)+M L^{2} \sin ^{2} \psi\right) \ddot{\phi} \\
& +2 M L^{2} \dot{\psi} \dot{\phi} \sin \psi \cos \psi=F_{\phi}
\end{aligned}
$$

The aforementioned equations can be transformed to nonlinear state space, by defining:

as the state variables:

$$
\theta, \dot{\theta}, \psi, \dot{\psi}, \phi, \dot{\phi}
$$

$$
X=\left[x_{1}, x_{2}, x_{3}, x_{4}, x_{5}, x_{6}\right]=[\theta, \dot{\theta}, \psi, \dot{\psi}, \phi, \dot{\phi}] .
$$

Hence, the general form of the state space is given as following equations:

$$
M(x) \dot{x}=f(x)+u
$$

where:

$$
M(x)=\left[\begin{array}{cccccc}
1 & 0 & 0 & 0 & 0 & 0 \\
M_{21} & M_{22} & -M_{24} & M_{24} & 0 & 0 \\
0 & 0 & 1 & 0 & 0 & 0 \\
-M_{21} & M_{24} & M_{21} & M_{44} & 0 & 0 \\
0 & 0 & 0 & 0 & 1 & 0 \\
0 & 0 & 0 & 0 & M_{65} & M_{06}
\end{array}\right]
$$




$$
\begin{gathered}
f(x)=\left[\begin{array}{l}
f_{1} \\
f_{2} \\
f_{3} \\
f_{4} \\
f_{5} \\
f_{6}
\end{array}\right]=\left[\begin{array}{c}
x_{2} \\
M L R x_{4}^{2} \sin x_{3}+2 T_{s} \\
x_{4} \\
M g L \sin x_{3}+M L^{2} x_{6}^{2} \sin x_{3} \cos x_{3} \\
x_{6} \\
-2 M L^{2} x_{4} x_{6} \sin x_{3} \cos x_{3}
\end{array}\right] \\
\mathrm{u}=\left[\begin{array}{c}
0 \\
u_{2} \\
0 \\
-u_{2} \\
0 \\
u_{6}
\end{array}\right]=\left[\begin{array}{cc}
0 & 0 \\
\frac{n k_{t}}{R_{m}} & \frac{n k_{t}}{R_{m}} \\
0 & 0 \\
-\frac{n k_{t}}{R_{m}} & -\frac{n k_{t}}{R_{m}} \\
0 & 0 \\
\frac{n k_{1} w}{2 R R_{m}} & -\frac{n k_{1} w}{2 R R_{m}}
\end{array}\right]\left[\begin{array}{l}
v_{r} \\
v_{l}
\end{array}\right]
\end{gathered}
$$

$\mathrm{u}$ is the control input which is generated by the controllers in this study.

\section{CONTROLLER DESIGN AND SIMULATION}

\section{A. State feedback control having $L Q R$}

To stabilize the two-wheel inverted pendulum, the optimal SFC have been designed using LQR. Following is the linear quadratic regulator objective function:

$J=\int_{0}^{\infty} x^{T}(t) Q x(t)+u^{T}(t) R u(t) d t$

The optimal control input to satisfy the above mentioned equation is:

$u(t)=-K x(t)$, where $K=R^{-1} B^{T} P$

$\mathrm{P}$ can be calculated by solving Riccati equation:

$A^{T} P+P A-P B R^{-1} B^{T} P+C^{T} C=0$

Where A, B and C are state space matrices. Two optimal LQRs have been designed for left and right DC motors.

There are two measurements of the angle from two different sources. The measurement from accelerometer gets affected by sudden horizontal movements (it could be used to measure the $\psi$ ) and the measurement from gyroscope gradually drifts away from actual value (it could be used to measure $\theta$ ). In other words, the accelerometer reading gets affected by short duration signals and the gyroscope reading is affected by long duration signals. To stabilize the robot, two state feedback controllers work simultaneously to control the states of DC motors.

The state feedback controller has been applied to the simulated model which has been presented in the Eq. (15). Figure 2 is the block diagram of closed-loop states control for the TWIP. The gains details are given as in TABLE 2.

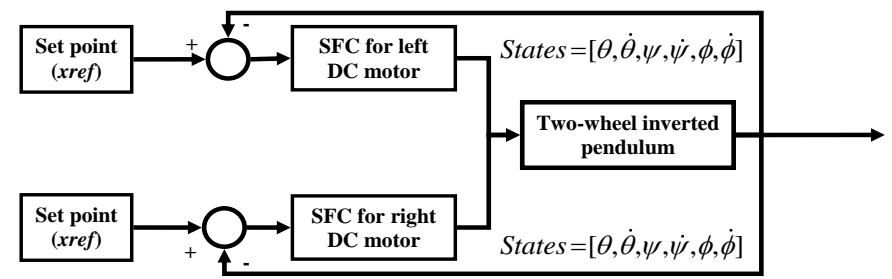

Figure 2. TWIP closed loop block diagram with LQR s xref: It represents the desired states, in this case to stabilize the TWIP the $x r e f=0$ rad (Figure 2).

\begin{tabular}{|c|cccccc|}
\hline & \multicolumn{5}{|c|}{$\left[k_{1}, k_{2}, k_{3}, k_{4}, k_{5}, k_{6}\right]$} \\
\hline $\begin{array}{c}\text { Left } \\
\text { motor }\end{array}$ & {$\left[\begin{array}{lllllll}-0.7071 & -0.3078 & -11.3966 & -1.6791 & 0.0000 & 0.2118\end{array}\right]$} \\
\hline $\begin{array}{c}\text { Right } \\
\text { motor }\end{array}$ & {$\left[\begin{array}{llllll}-0.7071 & -0.3078 & -11.3966 & -1.6791 & 0.0000 & -0.2118\end{array}\right]$} \\
\hline
\end{tabular}

The simulation of the closed-loop SFC has been done in SIMULINK using ode45 method with variable time step. As shown in Figure 3, two different initial pinch angles have been provided to evaluate the performance of LQR.
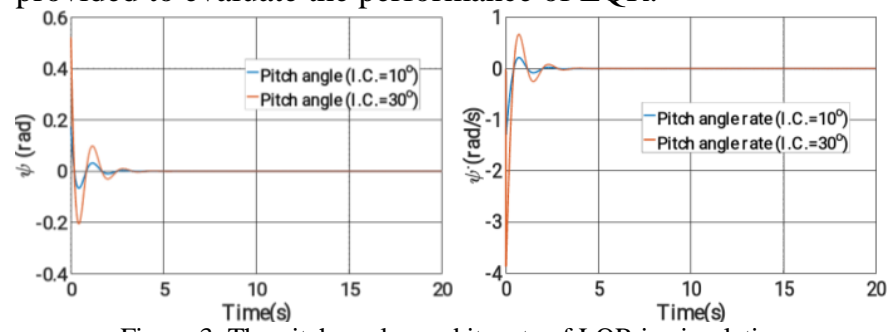

Figure 3. The pitch angle cand its rate of $L Q R$ in simulation

\section{A. Sliding mode controller}

As the yaw motion and self-balancing of the robot need to be controlled properly, a sliding mode control is proposed and designed to achieve self-balancing and stabilizing. Due to the system's nonlinearity and uncertainty, LQR could not precisely balance the pendulum and reject the large disturbance within short time period. Hence, the sliding mode controller is designed to handle the nonlinearity of the system using the sliding surface approach.

The design of the wheel angle sliding mode controller is presented as follows:

$$
\begin{aligned}
& \mathrm{s}_{1}=\mathrm{c}_{1} \psi+\mathrm{c}_{2} \dot{\psi} \\
& \dot{\mathrm{s}}_{1}=-\varepsilon_{1} \operatorname{sgn}\left(s_{1}\right) \\
& \mathrm{u}_{2}=\frac{c_{1}\left(M_{24}^{2}-M_{22} M_{44}\right) \dot{\psi}+\left(M_{24}^{2}+\mathrm{M}_{22} M_{44}\right) \varepsilon_{1} \operatorname{sgn}\left(s_{1}\right)}{c_{2}\left(-M_{24}-M_{22}\right)}+ \\
& \frac{C_{2} M_{24} f_{2}-C_{2} M_{22} f_{4}}{C_{2}\left(-M_{24}-M_{22}\right)}+ \\
& \frac{c_{2} M_{12}\left(-M_{24}+M_{22}\right) \dot{\theta}-\mathrm{C}_{2} M_{21}\left(M_{24}+\mathrm{M}_{22}\right) \dot{\psi}}{n}
\end{aligned}
$$

The design of the yaw motion sliding mode controller is presented as follows:

$$
\mathrm{s}_{2}=\mathrm{c}_{3} \phi+\mathrm{c}_{4} \dot{\phi}
$$

By substituting the equations to the robot dynamic, the second input can be calculated as:

$$
\mathrm{u}_{6}=\frac{M_{66} \varepsilon_{2} \operatorname{sgn}\left(s_{2}\right)}{-c_{4}}-f_{6}+\dot{\phi}\left(M_{65}-C_{3} M_{66}\right)
$$

where:

$$
\begin{aligned}
& \mathrm{f}_{2}=\operatorname{MLRx}_{4}^{2} \sin \mathrm{x}_{3}+2 \mathrm{~T}_{\mathrm{s}} \\
& \mathrm{f}_{4}=\operatorname{MgL\operatorname {sin}x_{3}}+\operatorname{ML}^{2} \mathrm{x}_{6}^{2} \sin \mathrm{x}_{3} \cos \mathrm{x}_{3}
\end{aligned}
$$




$$
f_{6}=-2 M L^{2} x_{4} x_{6} \sin x_{3} \cos x_{3}
$$

From Eq. 26 and Eq. 27, the inputs of left and right wheeels are:

$$
\begin{aligned}
& \mathrm{v}_{\mathrm{r}}=\frac{\mathrm{RR}_{\mathrm{m}}}{\mathrm{nk}_{\mathrm{t}} \mathrm{W}} \mathrm{u}_{6}-\frac{\mathrm{R}_{\mathrm{m}}}{2 \mathrm{nk}_{\mathrm{t}}} \mathrm{u}_{2} \\
& \mathrm{v}_{\mathrm{l}}=-\frac{\mathrm{RR}_{\mathrm{m}}}{\mathrm{nk}_{\mathrm{t}} \mathrm{W}} \mathrm{u}_{6}-\frac{\mathrm{R}_{\mathrm{m}}}{2 \mathrm{nk}_{\mathrm{t}}} \mathrm{u}_{2}
\end{aligned}
$$

Figure 4 depicts the block diagram of closed look position control with sliding mode controller for the TWIP.

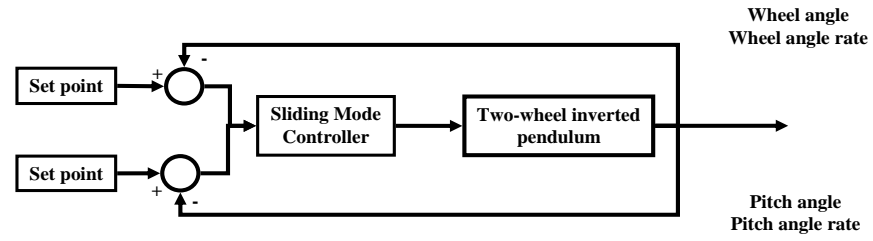

Figure 4. SMC block diagram for TWIP

The angular reaction of the wheels is presented in Figure 5:
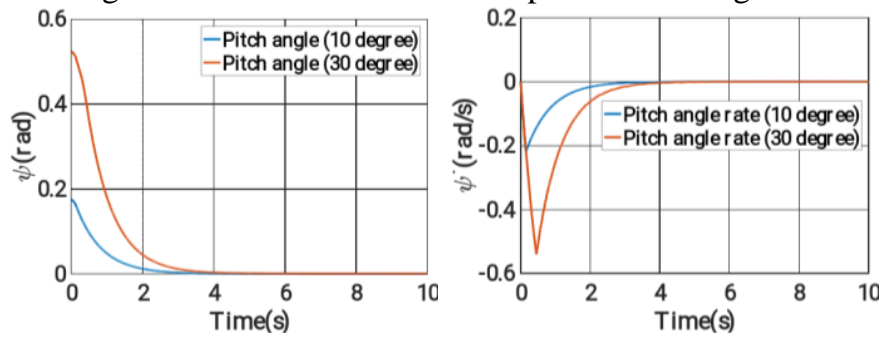

Figure 5. The pitch angle and its rate of SMC in simulation

TABLE 3. TRANSIENT PERFORMANCE OF SFC AND SMC IN SIMULATION

\begin{tabular}{|c|c|c|c|c|}
\hline & \multicolumn{2}{|c|}{ LQR } & \multicolumn{2}{c|}{ SMC } \\
\hline Initial condition & $10^{\circ}$ & $30^{\circ}$ & $10^{\circ}$ & $30^{\circ}$ \\
\hline Rise Time (s) & 0.181 & 0.166 & 3.144 & 4.374 \\
\hline Settling Time (s) & 2.25 & 3.04 & 1.638 & 2.581 \\
\hline $\begin{array}{c}\text { Percent of overshoot } \\
(\%)\end{array}$ & 36.12 & 38.36 & 0 & 0 \\
\hline
\end{tabular}

The simulation results are summarized in TABLE 3. From this table, one can see that the SMC has better transient performance than LQR does in terms of settling time and percent of overshoot.

\section{EXPERIMENT}

This TWIP robot is built with four main parts: controller and sensors, gear DC motors, battery, and structure. Arduino Uno is the controller of the TWIP and allows the driver shield to drive gear DC motors. The gear DC motors of the robot could robustly keep the robot stable. The driver shield is L298, which is a dual full-bridge driver. It can transform the real time data from Arduino board to the DC motors. To record the angular position like pitch, yaw and wheel angles, Arduino has been connected to two different sensors. (i.e., IPU 6050 which has accelerometer sensors, gyroscope). As it contains 16-bits analog to digital conversion hardware for each channel, it could be more precise.

In addition, using $\mathrm{X}$-bee shield can wirelessly provide the data transmission to computer. The gear-DC-motors made by Faulhaber with maximum revolution of $350 \mathrm{rpm}$. Moreover, the power is supplied by Li-Po battery/4000 mA. The diameter of wheels is $120 \mathrm{~mm}$. the center mass of robot is located in the middle of the wheels' axis.

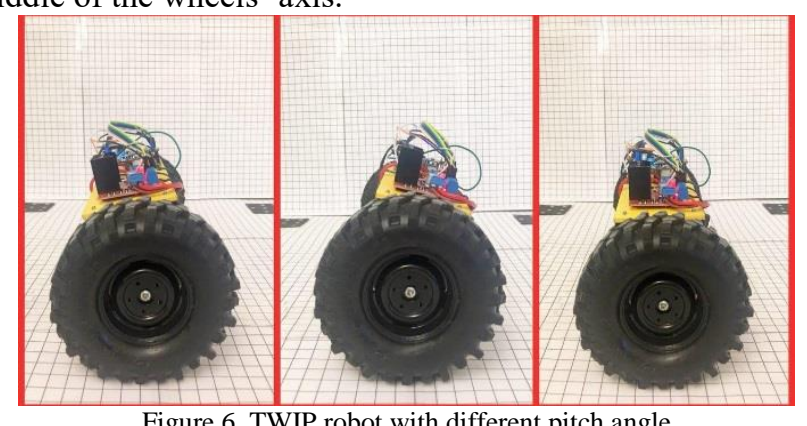

Figure 6. TWIP robot with different pitch angle

The experimental results of pitch angle and its rate of SFC controller are presented in Figure 7.
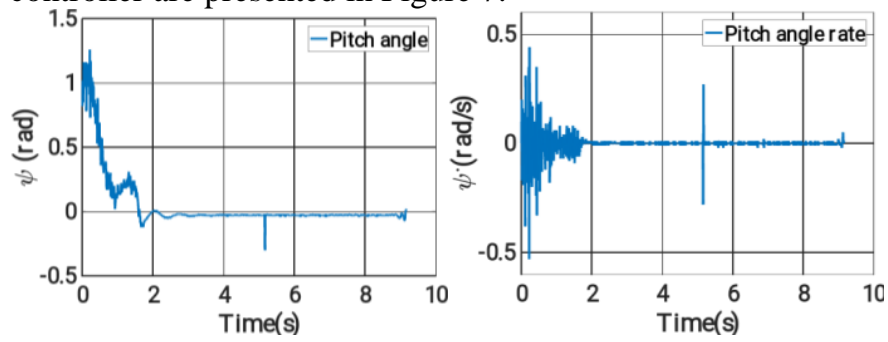

Figure 7. The experiment results of pitch angle and its rate of LQR In the next, to evaluate the performance of the sliding mode control, the pitch angle and its rate have been illustrated in Figure 8.
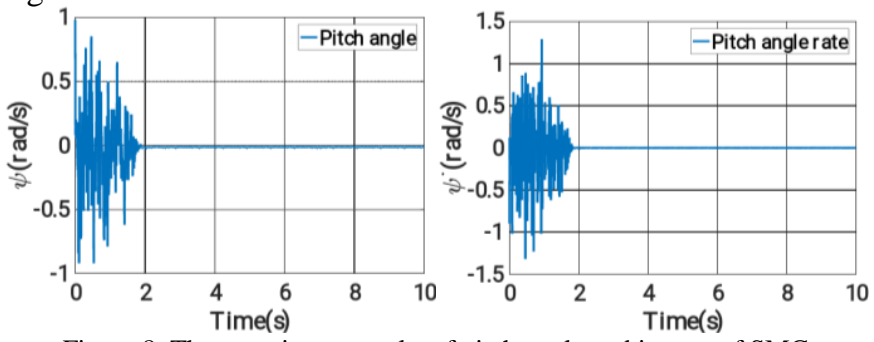

Figure 8. The experiment results of pitch angle and its rate of SMC

In both experimental tests, a big push has been applied to the TWIP robot around 1 second to test the disturbance rejection ability of both controllers. From Figs. 7 and 8, one can see that both SFC and SMC can recover from the push and achieve self-balancing. However, it takes SMC less than 2 seconds to settle in the zero angles position while SFC takes more than 2 seconds to reach the self-balancing state. The video of the experiments is uploaded in Youtube. https://youtu.be/a6w5zxU8IBU. The experimental tests demonstrate that SMC outperforms the LQR.

\section{CONCLUSION}

In this paper, a customer designed TWIP robot and it's modeling and control are presented. The ribot is an inherent 
unstable and nonlinear system, which poses challenge for control design. A SMC for balancing and steering movement is designed based on the dynamic model derived by the Lagrangian function method. From simulation results of the LQR and SMC for the TWIP robot, it can be concluded that the SMC has better transient performance in stabilizing the TWIP robot. To further evaluate the SMC and LQR performances, experimental tests have been conducted and validated the effectiveness of the designed controllers. As it is presented in the aforementioned test results, the performances of SMC is superior to that of LQR in terms of settling time and robustness. The future work includes the further improvement of control performances considering the actuation constraints.

\section{REFERENCES}

[1] Nasir, A. N. K., M. A. Ahmad, and RMT Raja Ismail. "The control of a highly nonlinear two-wheels balancing robot: A comparative assessment between LQR and PID-PID control schemes." World Academy of Science, Engineering and Technology 70 (2010): 227-232.

[2] VILLACRÉS, JUAN, MICHELLE VISCAINO, MARCO HERRERA, and OSCAR CAMACHO. "Controllers comparison to stabilize a twowheeled inverted pendulum: Pid, lqr and sliding mode control." moment 2, no. 2 (2016): 12.

[3] Marzi, Hosein. "Fuzzy control of an inverted pendulum using AC induction motor actuator." In Computational Intelligence for induction motor actuator. In Computational Intelligence for
Measurement Systems and Applications, Proceedings of 2006 IEEE Measurement Systems and Applications, Proceedings
International Conference on, pp. 109-114. IEEE, 2006.

[4] Unluturk, Ali, and Omer Aydogdu. "Adaptive control of two-wheeled mobile balance robot capable to adapt different surfaces using a novel artificial neural network-based real-time switching dynamic controller." International Journal of Advanced Robotic Systems 14, no. 2 (2017): 1729881417700893.

[5] Velazquez, Miguel, David Cruz, Salatiel Garcia, and Manuel Bandala. "Velocity and motion control of a self-balancing vehicle based on a cascade control strategy." International Journal of Advanced Robotic Systems 13, no. 3 (2016): 106.

[6] Gans, Nicholas R., and Seth A. Hutchinson. "Visual servo velocity and pose control of a wheeled inverted pendulum through partial-feedback linearization." In Intelligent Robots and Systems, 2006 IEEE/RSJ International Conference on, pp. 3823-3828. IEEE, 2006.

[7] Dai, Fuquan, Xueshan Gao, Shigong Jiang, Wenzeng Guo, and Yubai Liu. "A two-wheeled inverted pendulum robot with friction compensation." Mechatronics 30 (2015): 116-125. 